\title{
Conditioning of odors in compound with taste: A failure to observe potentiation
}

\author{
ROBERT A. ROSELLINI and ROBIN L. LASHLEY \\ State University of New York at Albany, Albany, New York
}

\begin{abstract}
In three experiments, rats were presented with odor-alone and compound odor-taste solutions, followed by lithium-chloride-induced illness. Subsequently, aversions to the odor stimuli were assessed. The results do not replicate earlier reports of potentiation of conditioning to odors by taste; greater aversion to the odor conditioned in compound, relative to that conditioned in isolation, was not evidenced for all the conditioned stimuli (CSs) employed. Instead, the outcome appeared to be odor-specific. This was consistently the case, despite manipulations of preexposure, type of taste CS employed, and intensity of odor CSs employed. These results suggest that the odor-taste potentiation phenomenon is more fragile than previously reported.
\end{abstract}

The amount of associative strength accruing to a conditional stimulus (CS) is a function of the simultaneous presence of other CSs (Rescorla \& Wagner, 1972). For example, Kamin (1969) has shown that a tone attains more associative strength if conditioned in isolation than if presented in compound with a light. This overshadowing phenomenon has received considerable attention in current theories of Pavlovian conditioning (e.g., Mackintosh, 1975; Rescorla \& Wagner, 1972).

The ubiquity of overshadowing has recently been questioned in research involving the taste aversion paradigm. Several investigators (Durlach \& Rescorla, 1980; Palmerino, Rusiniak, \& Garcia, 1980; Rusiniak, Hankins, Garcia, \& Brett, 1979) have reported that an odor CS followed by poison acquires little associative strength, but develops considerably more if conditioned in compound with taste. These demonstrations of potentiation (enhanced conditioning to a CS by virtue of being presented in compound with a stronger CS) are at odds with the overshadowing typically observed when exteroceptive CSs such as tones and lights are used.

These investigations do, however, contain conflicting evidence concerning the impact of prior experience. Palmerino et al. (1980) found that a single preexposure, either to the compound or to the odor and taste elements separately, reduces the amount of potentiation observed. In contrast, Durlach and Rescorla (1980) preexposed animals to the odor-taste compound and observed no such diminution. Palmerino et al. poisoned animals to the compound, then tested separately for aversions to odor and to taste; Durlach and Rescorla conditioned animals both to a compouund and to an isolated odor, then assessed potentiation by comparing intake of the odor elements in

This research was partially supported by NSF Grant No. BNS 7820678. We wish to thank Jeffery Carlson, Joseph P. DeCola, Mark Plonsky, Michael E. Abbot, and Anne Stilman for their assistance in this project. Requests for reprints should be sent to the second author at Kent State University, Tuscarawas Campus, New Philadelphia, $\mathrm{OH}$ 44663. a two-bottle test. It is not clear why preexposure had such differential effects when these alternate procedures were employed.

The purpose of the present studies was to further investigate the conditions under which prior experience affects potentiation. Given the absence of a preexposure effect in the Durlach and Rescorla study, we chose to employ their within-group procedures. It is possible that a single session, as in their Experiment 1, is not sufficient to interfere with potentiation, but that more prolonged preexposure may be effective.

\section{EXPERIMENT 1}

In this study, animals were exposed to an odor-taste (OT) compound and separately to an odor (O) for either 1 or 6 days. Subsequently, an aversion was conditioned to both the OT and O stimuli on 2 separate days. Subjects were then tested for intake of each odor using a twobottle test. Potentiation should be evidenced as lower consumption of the odor conditioned in compound, relative to that conditioned in isolation.

\section{Method}

Subjects. The subjects were 48 male Holtzman rats approximately 180 days old at the start of the study. In this and all subsequent experiments, animals had previously experienced inescapable shock and maintenance on an $85 \%$ body-weight food-deprivation schedule. At least 25 days prior to the start of the experiment, they were returned to adlib feeding. Treatments were carried out during the light phase of a 12hr light/dark cycle.

Apparatus. All experimental treatments were conducted in the animals' home cages $(48 \times 35 \times 35 \mathrm{~cm})$. Food was removed prior to each session, and the food hopper was used to hold the 100 -ml graduated cylinders in which solutions were presented.

As in Durlach and Rescorla (1980), the two odor CSs were $1.5 \% \mathrm{v} / \mathrm{v}$ solutions of Ann Page brand almond extract and Durkee brand banana extract, respectively, in distilled water; the taste stimulus was a $.06 \%$ $w / v$ solution of sodium saccharin in distilled water.

Procedure. Subjects were maintained on 10-min access to fluid intake per day for the duration of the experiment. For the first 6 days, they received distilled water. Subsequent treatment consisted of three phases: (1) preexposure, (2) conditioning, and (3) test. 
Preexposure. Half of the subjects were presented with the to-beconditioned stimuli for six sessions, while the remainder received water for the first 5 days and the solutions on Day 6. Within each condition, half of the subjects received banana-scented saccharin solution (BS) and almond-scented water (A); the other half received almond-scented saccharin solution (AS) and banana-scented water (B). Exposure consisted of 5-min access to each solution, separated by $5 \mathrm{~min}$. Order of exposure was counterbalanced, such that half of each group received OT followed by $\mathrm{O}$, and half received $\mathrm{O}$ followed by OT. In addition, ordering was alternated daily for the six-session preexposure condition: animals exposed to OT/O on Day 1 were given O/OT on Day 2, and so on, and the reverse for those first receiving O/OT.

Conditioning. On the 1 st conditioning day, each subject was given either the OT or O solution for $10 \mathrm{~min}$, followed $15 \mathrm{~min}$ later by a 5$\mathrm{ml} / \mathrm{kg}$ ip injection of $0.6 \mathrm{M}$ lithium chloride $(\mathrm{LiCl})$. Two days later, the alternate solution was presented, followed by $\mathrm{LiCl}$. A recovery session between the 2 poisoning days consisted of 10-min access to distilled water.

Aversion test. Two recovery days were given, followed by 2 days of measuring conditioned aversion using a two-bottle choice test. This consisted of $10 \mathrm{~min}$ of simultaneous presentation of each odor element. The initial bottle position for each solution was counterbalanced across subjects and alternated across the 2 days, and bottles were reversed midway through each session, to minimize any position preference effects.

\section{Results}

Figure 1 shows mean odor intakes as a function of preexposure. Groups BS/A consumed less of the bananascented water (B) which had been conditioned in compound than almond-scented water (A) conditioned in isolation, regardless of the number of preexposures. In Groups AS/B, however, although the 1-day preexposure animals consumed less A than B, this difference was considerably smaller, and the 6-day group actually drank more A.

The data were analyzed by means of group (BS/A or AS/B) $\times$ preexposure $(1$ or 6 days $) \times$ test odor $(A$ or B) analysis of variance. The main outcome was a significant three-way interaction $[F(1,44)=6.35, p<.015]$. Separate simple effects analyses were conducted for each group as a function of preexposure and test odor. Group BS/A showed only a significant effect of test odor $[F(1,22)$ $=13.33, p<.001]$ and no main effect of preexposure or any interaction with preexposure (all $p s>.30$ ). In contrast, Group AS/B showed a significant preexposure $\times$ test odor interaction $[F(1,22)=10.30, p<.005]$, but no main effect of either preexposure or test odor (all $p s>.20)$. As can be seen in the right-hand panel of Figure 1, the interaction results from the fact that animals in the single preexposure condition consumed less $A$ than $B$, and vice versa for those in the 6-day condition.

\section{Discussion}

These results are problematic, because they are obtained from a procedure virtually identical to that of Durlach and Rescorla (1980) but do not consistently demonstrate the occurrence of odor-taste potentiation. We did observe stronger rejection of banana odor conditioned in compound with taste than almond odor conditioned in isolation, regardless of the preexposure manipulation. However, in Group AS/B, animals given one preexposure showed no significant differences in intake, and those receiving six preexposures, in fact, displayed stronger rejection of the isolated banana odor. This outcome is in the direction of overshadowing, rather than the predicted potentiation.

This effect of odor type suggests that other factors are involved. One factor may be the particular taste employed; that is, saccharin may be

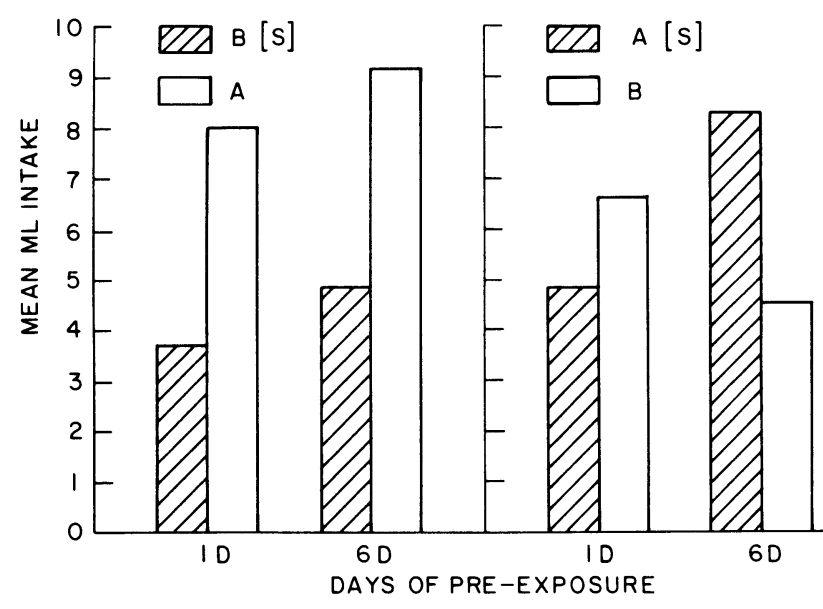

Figure 1. Mean milliliter intake of banana- and almond-scented water for Group BS/A (left panel) and AS/B (right panel) as a function of preexposure ( $B=$ banana, $A=$ almond, and $S=$ saccharin).

more capable of potentiating conditioning to banana than to almond. A second factor might be the relative saliencies of the odor stimuli; that is, if banana and almond differ in saliency, they could accrue differential amounts of associative strength. These possibilities are explored in Experiments 2 and 3, respectively.

\section{EXPERIMENT 2}

To assess the possibility that our failure to demonstrate potentiation across different odor stimuli stems partly from an interaction of the particular odors and taste employed, three different taste stimuli were tested.

\section{Method}

Subjects and apparatus. The subjects were 48 male Holtzman rats approximately 180 days old at the start of the experiment. The apparatus was the same as that of Experiment 1.

Proce dure. As before, the two $\mathrm{O}$ stimuli were $1.5 \% \mathrm{v} / \mathrm{v}$ solutions of almond and banana extracts in distilled water. The six OT compounds were combinations of these with either a $.06 \% \mathrm{w} / \mathrm{v}$ solution of sodium saccharin (Durlach \& Rescorla, 1980, Experiment 1), a $1.16 \%$ w/v solution of sodium chloride (Durlach \& Rescorla, 1980, Experiment 3), or a $.000034 \mathrm{M}$ solution of quinine monohydrochloride (Rescorla \& Freberg, 1978).

Procedures were the same as those of the 1-day preexposure condition of Experiment 1, with one exception. In this and the subsequent studies, animals were preexposed to either O or OT on Day 1, and the alternate solution on the following day, as in Durlach and Rescorla (1980).

\section{Results and Discussion}

Figure 2 shows the mean intake of the odor solutions across the 2 test days as a function of taste and type of OT compound. The taste employed in the OT compound seems to have had little effect in producing differential rejection of the two odors. Analysis of variance as a function of taste (saccharin, sodium chloride, or quinine), compound type (banana-taste or almond-taste), and test odor (A or B) showed no significant effects of taste or any significant interactions of taste with other factors (all ps $>$.15). Once again, when B was conditioned in compound, it was rejected more than was A conditioned in isolation $[F(1,37)=23.02, p<.001]$, regardless of the 
particular taste CS employed. However, when A was conditioned in compound, its consumption was equivalent to that of B conditioned in isolation $(p>.15)$.

\section{EXPERIMENTS 3A, B, AND C}

The results of Experiments 1 and 2 are consistent with the notion that banana may simply be a more salient odor than is almond. If so, then banana might accrue greater associative strength than almond through direct association with the US, regardless of the presence or absence of a taste CS (Rescorla \& Wagner, 1972). A second possibility is that potentiation and saliency both contribute to conditioning. Thus, when banana is in compound, it is subsequently rejected more than almond due both to potentiation and to its having formed a stronger direct association with the US. When almond is in compound, potentiation might likewise occur, but the increment in aversion may be masked by the strong aversion accruing to banana by virtue of the latter's higher saliency.

The purpose of this series of experiments was to determine whether saliency is indeed an important factor. Experiment $3 \mathrm{~A}$ employed progressively higher concentrations of almond odor in the AS compound, while holding that of banana constant, to try to obtain potentiation to almond. Experiment 3B attempted to reduce the potentiation observed to banana in the BS compound, by progressively decreasing its intensity while holding that of almond constant. Finally, Experiment $3 \mathrm{C}$ attempted to obtain potentiation to both odors by employing a high concentration of almond and a low concentration of banana.

\section{Method}

Subjects and Apparatus. Sixty-four male Holtzman rats served as subjects. Age, housing, deprivation conditions, and apparatus were the same as those described in the previous experiments.
Procedure. Methods were identical to those of Experiment 2. In Experiment $3 \mathrm{~A}$, three groups $(\mathrm{N}=8)$ were conditioned to the AS compound and separately to the B element. Concentration of the isolated B solution was held constant at $1.5 \% \mathrm{v} / \mathrm{v}$, while that of $\mathrm{A}$ in the compound increased from $1.5 \%$ to $2.0 \%$ to $3.0 \% \mathrm{v} / \mathrm{v}$, for Groups 1,2 , and 3 , respectively. In Experiment $3 \mathrm{~B}$, three groups $(\mathrm{N}=8)$ were conditioned to the BS compound and separately to the A element. Here the concentration of A remained constant at $1.5 \% \mathrm{v} / \mathrm{v}$, while that of $\mathrm{B}$ decreased from $1.5 \%$ to $1.0 \%$ to $0.75 \% \mathrm{v} / \mathrm{v}$, for Groups 1,2 , and 3, respectively. In Experiment 3C, the AS/B and BS/A conditions of Experiments 1 and 2 were replicated with the difference that a weak intensity of B $(0.75 \%$ $\mathrm{v} / \mathrm{v})$ and a strong intensity of $\mathrm{A}(3.0 \% \mathrm{v} / \mathrm{v})$ served as the odor stimuli.

\section{Results and Discussion}

The top portion of Figure 3 shows the mean intake of the odor stimuli on the two test days as a function of intensity for Experiment $3 \mathrm{~A}$. As before, no differential intake was observed with both concentrations at $1.5 \% \mathrm{v} / \mathrm{v}$. Raising the concentration of A relative to B had little effect. Analysis of variance as a function of group (3) $\times$ test odor (2) showed no significant main effects or interactions, even for the highest concentration of A (all ps $>.10)$.

The bottom portion of Figure 3 shows mean intakes for Experiment 3B. Subjects for whom the B intensity of the BS compound was $1.5 \%$ or $1.0 \% \mathrm{v} / \mathrm{v}$ showed more rejection of B than A (as with the BS/A groups of Experiments 1 and 2). However, at an intensity of $0.75 \% \mathrm{v} / \mathrm{v}$, equivalent intakes were observed. Analysis of variance as a function of group (3) $\times$ test odor (2) revealed only a significant effect of test odor $[F(1,20)=6.58, p=.018]$, indicating lower consumption of B. Further post hoc comparisons showed significantly lower intake of B than A for the first two groups $(p s<.05)$ and no significant differences for the third $(p>.10)$.

Figure 4 shows the mean intake of the two solutions for Experiment $3 \mathrm{C}$, in which the extreme values of the odor concentrations were employed. At the weakest con-

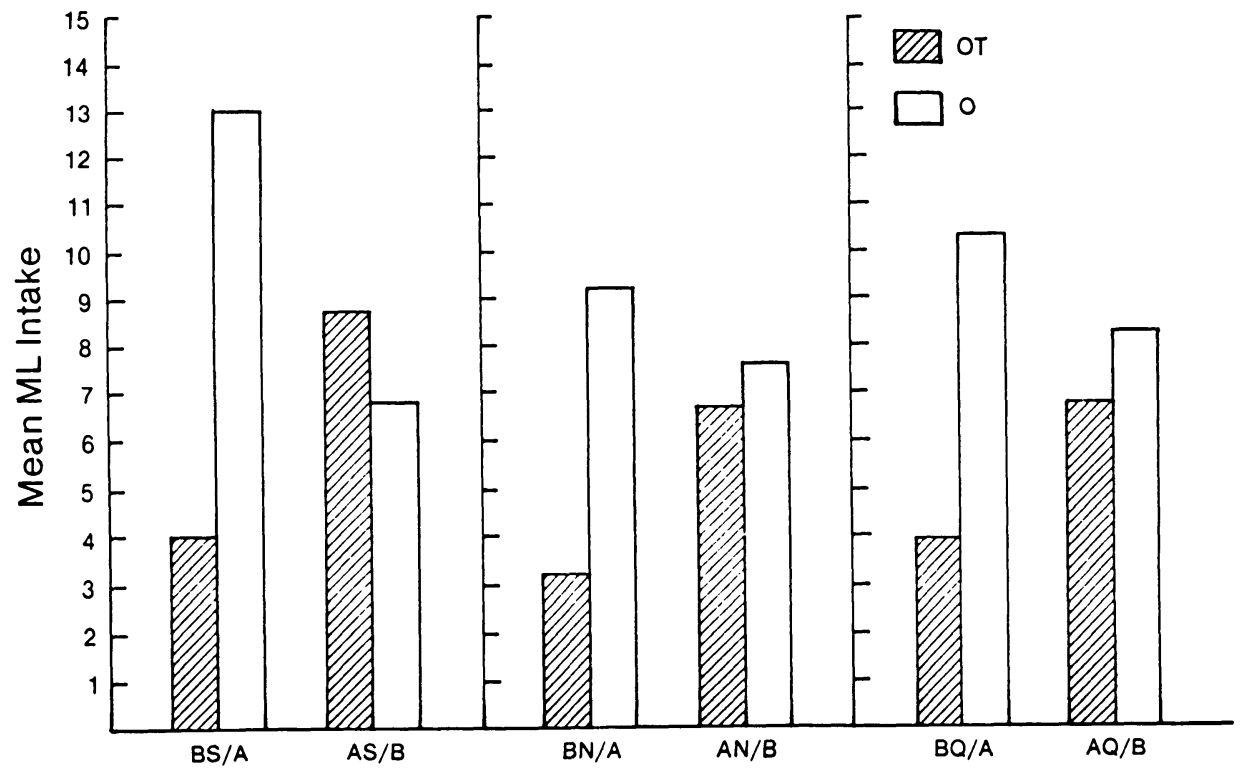

Figure 2. Mean milliliter intake of banana- and almond-scented water for each of the two conditioning groups as a function of taste ( $S$ =saccharin, left panel; $N$ = sodium chloride, middle panel; $Q$ = quinine, left panel). The hatched bars represent intake of the odor conditioned in compound with a taste. 
centration of $B$ conditioned in compound, there was more consumption of B than A; however, this difference was not statistically significant. With the strongest concentration of $\mathrm{A}$ in compound, again we observed no differential intake of the odors $(p s>.10)$.

In summary, decreasing banana intensity succeeded in reducing the extent of rejection accruing to it, consistent with a saliency hypothesis. However, even at the weakest $B$ and strongest A intensities we still did not observe potentiation to almond conditioned in compound.

\section{GENERAL DISCUSSION}

The results of these studies question the generality of the odor-taste potentiation phenomenon. In three experiments, we have not observed increased rejection of the odor of almond conditioned in compound with taste relative to banana conditioned in isolation. This is still the case if we employ different taste stimuli in the OT compound, as in Experiment 2, or different odor intensities, as in Experiment 3.

It should be noted that this difficulty in observing potentiation is not limited to our laboratory. Bouton and Whiting (1982) have failed to obtain potentiation of conditioning to almond odor using the Durlach and Rescorla (1980) procedures, as in the present studies, and they report overshadowing when using those of Rusiniak et al. (1979). Mikulka, Pitts,
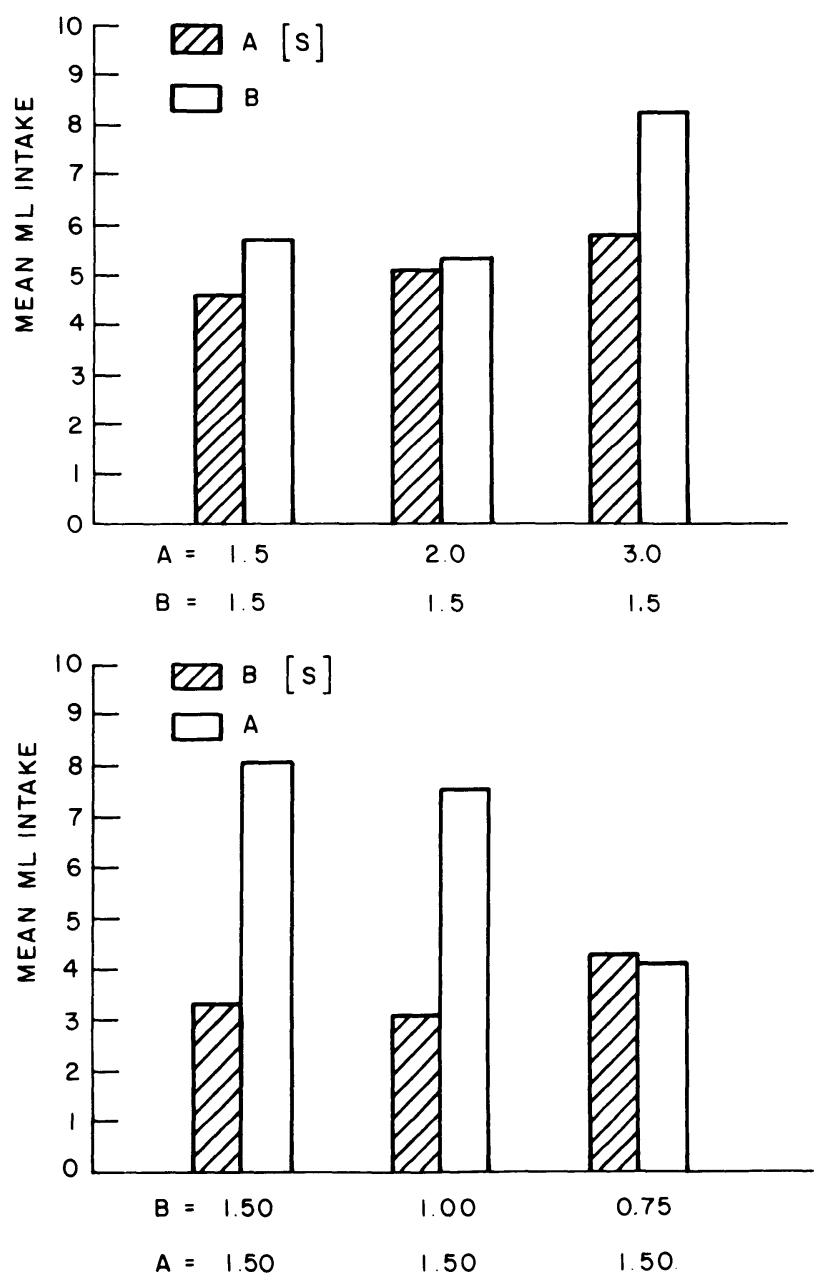

Figure 3. Mean milliliter intake of banana- and almond-scented water as a function of odor intensity in Experiments 3A (upper portion) and 3B (lower portion).

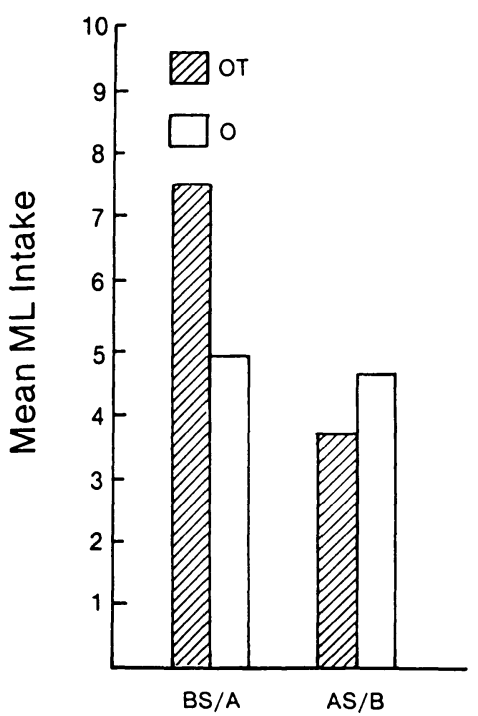

Figure 4. Mean milliliter intake of banana- and almond-scented water for the two conditioning groups. The hatched bars represent intake of the odor conditioned in compound with saccharin. The intensity of banana was $0.75 \% \mathrm{v} / \mathrm{v}$; the intensity of almond was $3.0 \% \mathrm{v} / \mathrm{v}$.

and Philput (1982), employing the Rusiniak et al. procedures, have also observed overshadowing rather than potentiation to almond odor, in studies in which the odor was conditioned in compound with either saccharin or sucrose. These findings, in conjunction with our own, suggest that the potentiation of conditioning to odor by taste may be limited to a fairly specific set of procedures and, as such, present less of a challenge to established theories of conditioning than has been recently proposed.

\section{REFERENCES}

Bouton, M. E., \& Whiting, M. R. (1982). Simultaneous odor-taste and taste-taste compounds in poison-avoidance learning. Learning \& Motivation, 13, 472-494.

Durlach, P. J., \& Rescorla, R. A. (1980). Potentiation rather than overshadowing in flavor-aversion learning: An analysis in terms of within-compound associations. Journal of Experimental Psychology: Animal Behavior Processes, 6, 175-187.

Kamin, L. J. (1969). Predictability, surprise, attention, and conditioning. In B. A. Campbell \& R. M. Church (Eds.), Punishment and aversive behavior. New York: Appleton-Century-Crofts.

Mackintosh, N. J. (1975). A theory of attention: Variations in the associability of stimuli with reinforcers. Psychological Review, 82, 276-298.

Mikulka, P. J., Pitts, E., \& Philput, C. (1982). Overshadowing not potentiation in taste aversion conditioning. Bulletin of the Psychonomic Society, 20, 101-104.

Palmerino, C. C., Rusiniak, K. W., \& Garcia, J. (1980). The peculiar roles of odor and taste in memory for poison. Science, 208, 753-755.

Rescorla, R. A., \& Freberg, L. (1978). The extinction of withincompound flavor associations. Learning \& Motivation, 9, 411-427.

Rescorla, R. A., \& Wagner, A. R. (1972). A theory of Pavlovian conditioning: Variations in the effectiveness of reinforcement and nonreinforcement. In A. H. Black \& W. K. Prokasy (Eds.), Classical conditioning II: Current theory and research. New York: AppletonCentury-Crofts.

Rusiniak, K. W., Hankins, W. G., Garcia, J., \& Brett, L. P. (1979). Flavor-illness aversions: Potentiation of odor by taste in rats. Behavioral \& Neural Biology, 25, 1-17.

(Manuscript received for publication September 28, 1985.) 